B Biol Sci. 2005;360(1461):1723-1737.

11. Knechtle SJ, Hamawy MM, Hu H, Fechner JH Jr, Cho CS. Tolerance and near-tolerance strategies in monkeys and their application to human renal transplantation. Immunol Rev. 2001;183:205-213.

12. Sacks S, Lee Q, Wong W, Zhou W. The role of complement in regulating the alloresponse. Curr Opin Organ Transplant. 2009;14(1):10-15.

13. Walters $\mathrm{S}$, et al. Increased CD4+Foxp $3+\mathrm{T}$ cells in BAFF-transgenic mice suppress $\mathrm{T}$ cell effector responses. J Immunol. 2009;182(2):793-801.

14. Bloom D, et al. BAFF is increased in renal transplant patients following treatment with alemtuzumab. Am J Transplant. 2009;9(8):1835-1845.

15. Larsen CP, et al. Rational development of LEA29Y (belatacept), a high-affinity variant of CTLA4-Ig with potent immunosuppressive properties. AmJ Transplant. 2005;5(3):443-453.
16. Li Y, Ma L, Yin D, Shen J, Chong AS. Long-term control of alloreactive $B$ cell responses by the suppression of T cell help. J Immunol. 2008;180(9):6077-6084.

17. Quezada SA, Jarvinen LZ, Lind EF, Noelle RJ. CD40/ $\mathrm{CD} 154$ interactions at the interface of tolerance and immunity. Annu Rev Immunol. 2004;22:307-328.

18. Ferguson SE, Han S, Kelsoe G, Thompson CB. CD28 is required for germinal center formation. J Immunol. 1996;156(12):4576-4581.

\title{
Pathogen-specific antibodies: codependent no longer
}

\author{
Edward N. Janoff',2 and Daniel N. Frank ${ }^{1,3}$
}

\begin{abstract}
${ }^{1}$ Mucosal and Vaccine Research Program Colorado. ${ }^{2}$ University of Colorado Denver School of Medicine, Department of Medicine, Division of Infectious Diseases, Aurora, and Department of Veterans Affairs Medical Center, Denver. ${ }^{3}$ Department of Molecular, Cellular, and Developmental Biology, University of Colorado Boulder.
\end{abstract}

\begin{abstract}
Antibody-mediated defense against pathogens typically requires complex interactions between antibodies and other constituents of the humoral and cellular immune systems. However, recent evidence indicates that some antibodies alone can inhibit pathogen function in the absence of complement, phagocytes, or NK cells. In this issue of the JCI, McClelland et al. have begun to elucidate the molecular bases by which antibodies alone can impact pathogen growth and metabolism. They show that mAbs specific for the polysaccharide capsule of the human pathogenic fungus Cryptococcus neoformans elicit diverse effects on fungal gene expression, lipid biosynthesis, susceptibility to amphotericin B, cellular metabolism, and protein phosphorylation. These data suggest that pathogens have the capacity to generate broad metabolic responses as a result of surface binding by pathogen-specific antibodies, effects that may hold therapeutic promise.
\end{abstract}

\section{Evolving concepts of antibody defense}

The word immunity derives from the Latin "immunitas" meaning exemption and has come to mean protection from disease. Immunity has been observed over the centuries during plagues in Athens and Byzantium, epidemics of bubonic plague and smallpox, as well as with snake bites and vaccinations (1). The identification of specific pathogens in the late 19th century was associated with the concept that such organisms were ultimately inhibited by depleting their environment of required nutrients, by their own metabolic by-products, or by the inhospitableness of infected tissues. Enter host defense. Initial conflicts arose between advocates of a predominantly soluble or humoral basis for immunity and those favoring a cellular basis. These disparate viewpoints were

Conflict of interest: Edward N. Janoff's laboratory has received research funding from Pfizer Pharmaceuticals (previously Wyeth Vaccines) and VaxInnate Corporation for vaccine studies unrelated to Cryptococcus.

Citation for this article: J Clin Invest. 2010; 120(4):1039-1042. doi:10.1172/JCI42695. ultimately reconciled in large part when antibodies, the key mediators of humoral immunity, were shown to rely on other soluble factors, particularly complement, and cells known as phagocytes to provide protection against and mediate resolution of infection. For its part, the microbe itself often expresses a range of protective defenses. These microbial virulence factors may bind, mask, or degrade complement components; cleave adherent antibodies (e.g., IgA1 protease); or subvert the activity of antibodies by binding to their effector Fc constant regions (e.g., via staphylococcal protein A or streptococcal protein $\mathrm{G}$ ) that otherwise direct pathogens to an $\mathrm{Fc}$ receptor-bearing phagocyte. The protective effects of antibodies are classically mediated through their specificity for the pathogen (facilitated via their variable regions) and the ability of their Fc constant region to act as a bridge or scaffold. Other host defense mechanisms (e.g., complement, phagocytes, and NK cells) use this foundation to induce the fatal injuries on the pathogen, on which antibody defense is dependent (Figure 1A).
However, in their study in this issue of the JCI, McClelland et al. advance an intriguing conceptual paradigm that binding of specific antibodies alone can elicit a range of metabolic perturbations in the fungal pathogen Cryptococcus neoformans (2). C. neoformans, widespread in the environment, is well-controlled and rarely symptomatic in healthy individuals, in large part because of antibody-dependent mechanisms. However, the organism causes recalcitrant disease and high mortality in patients with advanced cell-mediated immunodeficiency, such as those individuals with HIV/AIDS who have very low $\mathrm{CD}^{+}$ $T$ cell counts and patients who have undergone solid organ transplantation $(3,4)$. McClelland and colleagues show that three antibodies that bind to distinct topological sites on the polysaccharide capsule of C. neoformans elicit varying effects on its gene expression (2). The effects are direct and due to the antibodies in the absence of other soluble or cellular host elements, providing evidence that pathogens can recognize and respond to antibody binding by modulating distinct microbial genetic pathways (Figure 1B). These findings raise the intriguing possibility that the physiology of a pathogen and its susceptibility to clearance may be manipulated by rational antibody design.

\section{Building on the past}

Previous studies have revealed that, independent of the presence of complement or phagocytes, antibody-pathogen interactions can disrupt microbial integrity, although the genetic mechanism(s) remained undetermined (5-14). Antibodies 

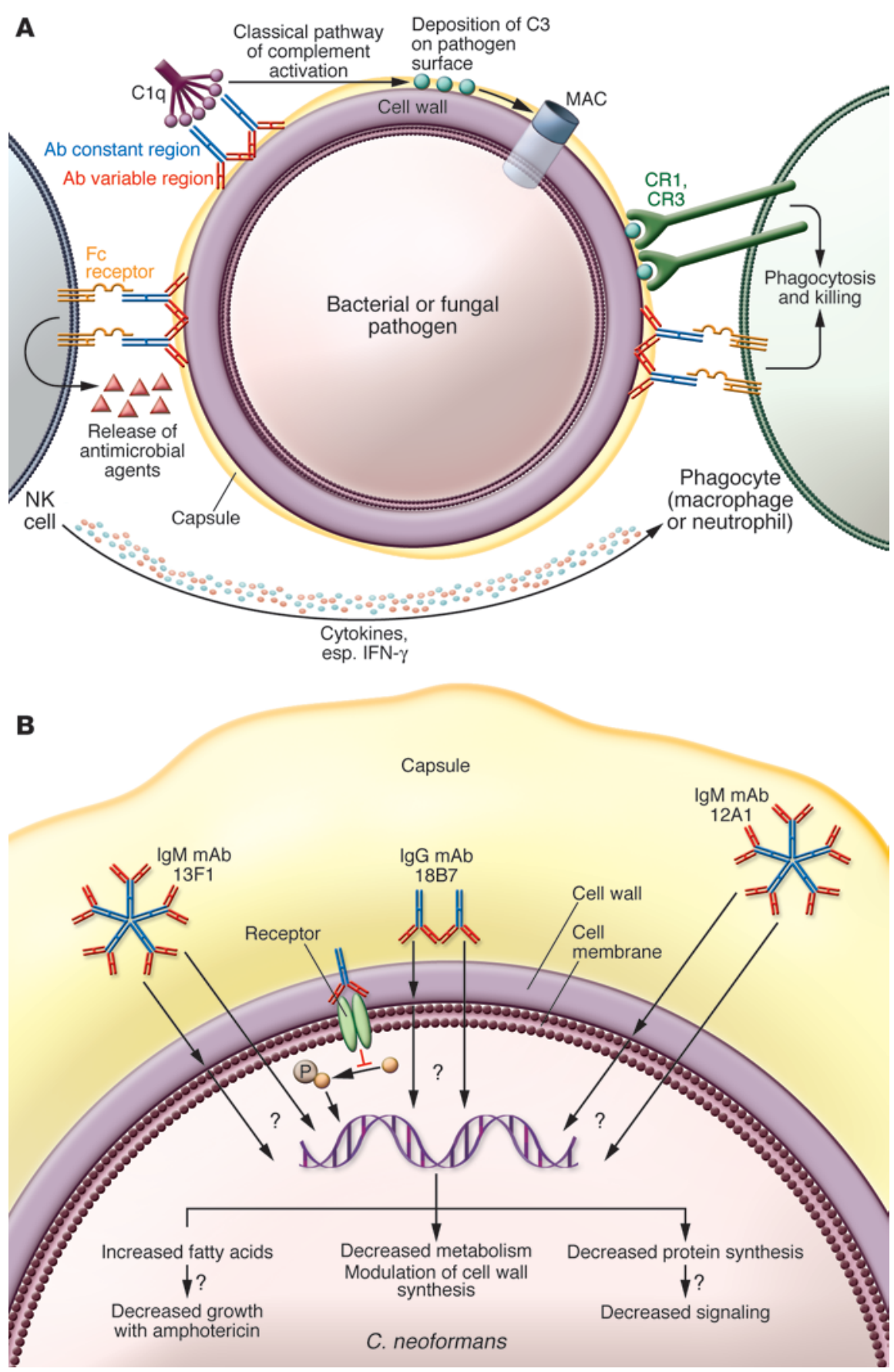

raised in mice against several pathogenic species of bacteria (e.g., Borrelia spp.) (5-9) and fungi (e.g., Candida, Fonsecaea, and Cryptococcus species; refs. 10-14) exhibit complement-independent microbicidal (i.e., fatal to microbes) or microbistatic (i.e., growth inhibiting) activities. IgM antibodies to surface-exposed antigens facilitated effective clearance of the Borrelia species in mice, in conjunction with direct injury to the outer bacterial membrane, but internal events were not examined. A human recom- binant mAb specific for HSP90 provided broad-spectrum growth inhibition of Candida species and improved the clinical and microbiological outcome of invasive candidiasis in both a murine model (11) and human patients (12) when coadministered with amphotericin B. However, the specific biochemical and/or physiological events underlying these additive effects have not been characterized. Did the complementary effects of the HSP90-specific antibody derive from structural effects on target cells

\section{Figure 1}

A pathogen's view of humoral immune defense. (A) Pathogen-specific antibody typically mediates its effects through the ability of its $\mathrm{Fc}$ constant region to act as a bridge to other host defense mechanisms (e.g., complement, phagocytes, and NK cells). Recognition of Fc by these immune components induces the fatal injuries to the pathogen, on which antibody defense is dependent. Cytotoxic processes include complement-dependent assembly of transmembrane pores (membrane attack complexes [MAC]), engulfment by phagocytes (macrophage or neutrophil), and release of antimicrobial agents by NK cells. CR1, complement receptor 1. (B) Possible direct effects of specific antibody on pathogen activity. The work of McClelland et al. (2) suggests multiple pathways by which antibodies may act on their target microbes in the absence of other immune factors. The diagram shows a cross-section of the human fungal pathogen $C$. neoformans, including the polysaccharide capsule that surrounds the fungal cell wall. In this study, mAb binding to both the outer (IgM) and inner (IgG) regions of the $C$. neoformans capsule triggered different genetic pathways and diverse, concomitant changes in fungal physiology and metabolism. Arrows denote hypothetical signaling pathways, currently undefined, which inform C. neoformans of the presence of the capsule-bound mAb and thus alter gene expression patterns. McClelland et al. report myriad responses to $\mathrm{mAb}$ binding, including upregulation of fatty acid-synthesis genes, activation of lipid biosynthesis, reduced cellular metabolism, reduced expression of protein synthesis genes, diminished protein phosphorylation, and increased sensitivity to the antifungal drug amphotericin B. Further elucidation of the biochemical and cell-biological consequences of antibody binding may lead to rational design of microbicidal antibodies. by disrupting cell wall-bound transporters and essential membrane structures? Alternatively, could antibody binding have induced signaling or disruption of signal transduction pathways, thereby interfering with critical gene expression? The work of McClelland et al. begin to address, for the first time to our knowledge, the impact of surface-bound antibodies on gene expression and suggests mechanisms that may underlie the interaction of antibodies and antifungal agents. 


\section{Genetic bridge to the future}

McClelland and colleagues undertook an in vitro systems biology analysis of the biochemical and biological effects mediated by $\mathrm{mAb}$ binding to the polysaccharide capsule of C. neoformans (2). An important first experiment assessed whether binding of the capsule-specific mAbs to C. neoformans cells induced alterations in gene expression, as assessed by microarray analysis of mRNA. Three C. neoformans capsule-specific mAbs were tested, one IgG mAb (18B7) and two IgM mAbs (12A1 and $13 \mathrm{~F} 1$ ), along with nonspecific isotypematched control mAbs. Relative to the control antibodies, each specific mAb elicited a different pattern of mRNA induction or repression. IgG $\mathrm{mAb} 18 \mathrm{~B} 7$ upregulated or downregulated 43 genes, including several encoding proteins involved in fattyacid biosynthesis. In contrast, mAb 12A1 altered expression of 62 genes, including downregulation of 8 genes encoding proteins involved in ribosome biogenesis and translation. Quantitative reverse-transcription PCR confirmed the microarray results in most but not all cases.

The antibody-altered gene expression patterns detected by McClelland and colleagues suggest interference with biochemical pathways involved in lipid metabolism and/or cell wall synthesis (2), hypotheses supported by several follow-up experiments. First, fungal growth kinetics revealed that the IgG $\mathrm{mAb} 18 \mathrm{~B} 7$ enhanced the potency of the antifungal agent amphotericin B, which acts through association with cell membrane ergosterol. Compared with amphotericin $\mathrm{B}$ alone, addition of a half-saturating concentration of $\mathrm{mAb} 18 \mathrm{~B} 7$ delayed C. neoformans growth. How a control mAb also delayed growth, albeit to a lesser extent, as well as whether the effects on growth were present in the absence of amphotericin B may require further elucidation. Second, binding of $\mathrm{mAb} 18 \mathrm{~B} 7$ induced higher levels of lipids, including ergosterol, relative to the nonspecific mAb control. Based on these data, the authors propose that the synergy between amphotericin $\mathrm{B}$ and $\mathrm{mAb}$ 18B7, in depressing C. neoformans growth rates, resulted from $\mathrm{mAb}$-induced accumulation of amphotericin B binding sites. This model is reminiscent of other cases in which antibodies binding to a polysaccharide capsule alter the surface of the pathogen and host-pathogen interactions under nonopsonizing conditions to the benefit (15) or detriment of the host (16). In the current study (2), the authors took the next step by identifying pathogen-specific gene expression. Finally, mAb-induced reductions in the steady-state levels of protein phosphorylation suggest that intracellular signalling pathways are disrupted by mAb 18B7, specifically those proposed to involve regulation of clathrin-mediated endocytosis.

\section{Translating transcripts into treatment?}

Overall, the work of McClelland and colleagues (2) provides provocative evidence of what seems to be a novel process, by which antibodies alone may modulate pathogens. A promising narrative framework has been established, providing others with the opportunity to add to the story. Does mAb binding directly impinge on intracellular signaling and gene expression, for example, through specific interactions with membrane-bound signal transduction proteins? Are the observed molecular phenotypes secondary responses to $\mathrm{mAb}$ binding, as might be expected if $\mathrm{mAb}$ binding were to disrupt structural components at the fungal cell surface, although the antibodies bound at different depths? Do these mAbs act as "catalytic antibodies," such as those described by Wentworth and colleagues (17), that generated antibody-associated oxidant-mediated injury to Escherichia coli in a process proposed to be effected by $\mathrm{H}_{2} \mathrm{O}_{2}$, an ozone-like compound, and ${ }^{\bullet} \mathrm{OH}$ hydroxyl radicals (18). In the current study, the source of substrate for generating such products is not clear.

Can the processes revealed in this in vitro study by McClelland and colleagues (2) translate into clinically relevant in vivo effects? Although not an element of normal host microbiota, environmental exposure to C. neoformans appears to be common. Results of serologic data, skin test responsiveness, and lymphocyte proliferation assays suggest high rates of asymptomatic but immunogenic infections over time in healthy individuals. Mechanisms of defense may involve direct interactions between the organism and $\mathrm{CD}^{+}$and $\mathrm{CD}^{+} \mathrm{T}$ cells, but much of the protection in healthy individuals likely derives from successful clearance of transient C. neoformans infection by specific antibody in conjunction with complement, phagocytes, and NK cells. However, each of these supportive immune components may be insufficient in patients with advanced cell-mediated immunodeficiency and during the most morbid and frequently fatal manifestation of cryptococcal disease, meningitis.
Indeed, Park, et al. estimate that up to a million cases of meningitis due to C. neoformans occur annually, primarily in patients with AIDS, among whom more that 600,000 die within the first three months (3). In this context, deaths from C. neoformans appear to exceed those from tuberculosis. The profile of the cerebrospinal fluid in most cases reveals limited inflammation, characterized by a low number of white cells (either lymphocytes or phagocytes) and low levels of protein, and complement levels that are typically quite low. Treatment with amphotericin B is only moderately effective ( $\leq 50 \%$ survival in Africa), and therapy with oral antifungal agents is most often continued for life. Similarly, among solid organ transplant patients, mortality with cryptococcal meningitis ranges from $20 \%$ to $50 \%$ (4). In these settings, passive administration of capsule-specific mAbs that impair the organism, without requiring effective contributions from complement, phagocytes, or NK cells in the cerebrospinal fluid (i.e., antibodies with the properties of those identified by McClelland and colleagues; ref. 2), early in the course of therapy, may provide a useful and much needed adjuvant to current therapy. That activity of the capsule itself limits the production of specific antibody by B cells further enhances the potential therapeutic contributions of passive immunization.

\section{Where to go now?}

The current study suggests that C. neoformans has the capacity to generate a broad metabolic, if not specifically cytotoxic, response to binding by pathogen-specific antibodies. The responses were not stereotypic, suggesting that the effects were not due to agglutination or nutritional deficits. Moreover, the authors did not observe substantial changes in genes encoding proteins directly associated with pathogenicity, such as those regulating the capsule or melanin production, although those controlling phospholipase production were affected. Of potential clinical relevance, growth of the fungus in the presence of the antifungal agent amphotericin B, which associates with ergosterol in the lipid membrane, was further impaired in vitro in the presence of one specific antibody. These data are presented by the authors in the context of dysregulation of membrane lipid synthesis. Thus, McClelland and colleagues (2) have taken us on a journey beyond surface phenotype to the central workings of the cell. Building on the genomic information of 
C. neoformans (19), they have intriguingly revealed that surface binding can modulate fungal metabolism in diverse ways. They indicate that, as observed by others with a range of bacteria, antibodies alone can have direct effects on microbes. Antibodies acting alone provide one more recognized mechanism of host defense, actions that may be most effective clinically in selected environments, such as the cerebrospinal fluid, where other host defense factors are limiting. Nevertheless, effective immunologic control of infection is best achieved when all relevant components are cooperative, even if individual constituents, such as specific antibodies, can stand alone.

\section{Acknowledgments}

This work supported by the Mucosal and Vaccine Research Program Colorado, University of Colorado School of Medicine, the Veterans Affairs Research Service, and NIH grant A1-077069.

Address correspondence to: Edward N. Janoff, Mucosal and Vaccine Research Program Colorado, University of Colorado Denver, RC-2, Box B-168, 12700 E. 19th Ave., Aurora, CO 80045. Phone: 303.724.4936; Fax: 303.388.1878; E-mail: Edward.Janoff@ucdenver.edu.
1. Silverstein AM. A History of Immunology. San Diego, CA: Academic Press, Inc; 1989.

2. McClelland EE, Nicola AM, Prados-Rosales R, Casadevall A. Ab binding alters gene expression in Cryptococcus neoformans and directly modulates fungal metabolism. J Clin Invest. 2010;120(4):1355-1361.

3. Park BJ, Wannemuehler KA, Marston BJ, Govender N, Pappas PG, Chiller TM. Estimation of the current global burden of cryptococcal meningitis among persons living with HIV/AIDS. AIDS. 2009; 23(4):525-530.

4. Singh N, Dromer F, Perfect JR, Lortholary O. Cryptococcosis in solid organ transplant recipients: current state of the science. Clin Infect Dis. 2008; 47(10):1321-1327.

5. Coleman JL, Rogers RC, Benach JL. Selection of an escape variant of Borrelia burgdorferi by use of bactericidal monoclonal antibodies to OspB. Infect Immun. 1992;60(8):3098-3104.

6. Coleman JL, Rogers RC, Rosa PA, Benach JL. Variations in the ospB gene of Borrelia burgdorferi result in differences in monoclonal antibody reactivity and in production of escape variants. Infect Immun. 1994;62(1):303-307.

7. Connolly SE, Thanassi DG, Benach JL. Generation of a complement-independent bactericidal IgM against a relapsing fever Borrelia. J Immunol. 2004; 172(2):1191-1197.

8. Escudero R, Halluska ML, Backenson PB, Coleman JL, Benach JL. Characterization of the physiological requirements for the bactericidal effects of a monoclonal antibody to OspB of Borrelia burgdorferi by confocal microscopy. Infect Immun. 1997; 65(5):1908-1915.

9. Sadziene A, Jonsson M, Bergstrom S, Bright RK, Kennedy RC, Barbour AG. A bactericidal antibody to Borrelia burgdorferi is directed against a variable region of the OspB protein. Infect Immun. 1994; 62(5):2037-2045.

10. Alviano DS, et al. Melanin from Fonsecaea pedro- soi induces production of human antifungal antibodies and enhances the antimicrobial efficacy of phagocytes. Infect Immun. 2004;72(1):229-237.

11. Matthews RC, et al. Preclinical assessment of the efficacy of mycograb, a human recombinant antibody against fungal HSP90. Antimicrob Agents Chemother. 2003;47(7):2208-2216.

12. Pachl J, et al. A randomized, blinded, multicenter trial of lipid-associated amphotericin B alone versus in combination with an antibody-based inhibitor of heat shock protein 90 in patients with invasive candidiasis. Clin Infect Dis. 2006;42(10):1404-1413.

13. Rodrigues ML, et al. Human antibodies against a purified glucosylceramide from Cryptococcus neoformans inhibit cell budding and fungal growth. Infect Immun. 2000;68(12):7049-7060.

14. Rosas AL, Nosanchuk JD, Casadevall A. Passive immunization with melanin-binding monoclonal antibodies prolongs survival of mice with lethal Cryptococcus neoformans infection. Infect Immun. 2001; 69(5):3410-3412.

15. Taborda CP, Casadevall A. CR3 (CD11b/CD18) and CR4 (CD11c/CD18) are involved in complement-independent antibody-mediated phagocytosis of Cryptococcus neoformans. Immunity. 2002; 16(6):791-802.

16. Weiser JN, Bae D, Fasching C, Scamurra RW, Ratner AJ, Janoff EN. Antibody-enhanced pneumococcal adherence requires IgA1 protease. Proc Natl Acad Sci U S A. 2003;100(7):4215-4220.

17. Wentworth P Jr, et al. Evidence for antibody-catalyzed ozone formation in bacterial killing and inflammation. Science. 2002;298(5601):2195-2199.

18. Nathan C. Immunology. Catalytic antibody bridges innate and adaptive immunity. Science. 2002; 298(5601):2143-2144.

19. Idnurm A, Bahn YS, Nielsen K, Lin X, Fraser JA, Heitman J. Deciphering the model pathogenic fungus Cryptococcus neoformans. Nat Rev Microbiol. 2005;3(10):753-764. 